\title{
Numerical Simulation of Non-Equilibrium Stationary Cur- rents Through Nano-scale One-Dimensional Chains
}

\author{
João Pedro dos Santos Pires ${ }^{1,2, *}$, Bruno Amorim ${ }^{1,3}$, and João Manuel Viana Parente Lopes ${ }^{1,2}$ \\ ${ }^{1}$ Centro de Física das Universidades do Porto e Minho \\ ${ }^{2}$ Faculty of Sciences, University of Porto, Rua do Campo Alegre 4169-007, Porto, Portugal \\ ${ }^{3}$ University of Minho, Campus of Gualtar, 4710-057, Braga, Portugal
}

\begin{abstract}
Using a method based on the time-evolution of the occupied states at zero temperature, we observe the onset of a quasi-uniform and quasisteady state current across a disordered tight-binding chain, coupled between two finite (but large) clean leads with open boundaries. This current is seen to match the one obtained in the Landauer-Büttiker formalism and is also independent of the initial condition considered (partitioned or non-partitioned). Finite-size effects are also reported and briefly discussed.
\end{abstract}

\section{Introduction}

Electron transport in solid-state systems has always been an important topic in condensed matter theory due to both its technological importance and the interesting quantummechanical effects it displays. In bulk materials, electron transport due to a static electric field is usually described in by a local conductivity, $\sigma_{D C}$. From the theoretical side, this local conductivity is computed using different Kubo formulas (KF) [6], for which several analytical and very efficient numerical methods have been developed $[1,4,5,10,16]$.

With the advent of nano-scale devices in the 1980's, the need for a different approach to this problem emerged. At low temperatures and for tiny samples, electrons propagate as coherent matter waves which are highly affected by the specific distribution of impurities and the presence/geometry of the electric contacts used in the experiment. In such situations, describing transport by a local conductivity is inadequate (see Ref. [14] for details). These issues were eventually settled by the application of a different formalism: the LandauerBüttiker formalism (LBF).

The $\operatorname{LBF}[2,7]$ recasts quantum electron transport as a wave scattering problem. The current that flows across a sample is described in terms of the transmission of incoming waves that are partially reflected and partially transmitted by the sample. These incoming waves originate from perfect and semi-infinite leads, which simulate the electrodes in an experiment. It is also assumed that the occupation of these propagating states is controlled by the distinct chemical potential of each lead. In the LBF, the current through the sample, at zero temperature, is given by the Landauer Formula $[8,17]$ :

$$
I_{\mathrm{DC}}=\frac{e}{h} \int_{E_{F}-e \frac{\Delta V}{2}}^{E_{F}+e \frac{\Delta V}{2}} d E \mathcal{T}(E),
$$

\footnotetext{
*e-mail: up201201453@fc.up.pt
} 
where $\mathcal{T}(E)$ is the transmission function, $E_{F} \pm \Delta V / 2$ is the chemical potential of each lead, with $\Delta V$ the potential bias that is applied between the two leads and $E_{F}$ a reference chemical potential. Despite its simple appearance, the Eq. (1) describes a fully non-linear DC current, where the integrand can be identified with the quantum transmittance, $\mathcal{T}$, of the sample at energy $E$. For small bias, the Landauer Formula reduces to $I_{\mathrm{DC}}=G_{\operatorname{lin}} \Delta V$, where $G_{\text {lin }}$ is the linear conductance, which is a function of $E_{F}$, and is proportional to the transmission:

$$
G_{\text {lin }}\left(E_{F}\right)=\frac{e^{2}}{h} \mathcal{T}\left(E_{F}\right)
$$

It must be pointed out that the Landauer formula only describes the steady-state current and requires the assumption that the leads have well-defined chemical potentials, which are ill defined out of equilibrium.

The issue of how this steady-state is achieved and if it even exists has been a subject of inquiry over the years $[3,13]$. When trying to address this problem, the question of what is the initial condition of the system, becomes relevant. It is therefore natural to consider two different initial conditions:

- Partitioned Setup: In which the leads and sample are initially disconnected, each being in a local equilibrium state described by a chemical potential and subject to a potential bias. The leads and sample are then brought suddenly into contact;

- Non-Partitioned Setup: A more realistic scenario, in which the leads and sample are initially connected and in equilibrium, with a common chemical potential. Then a bias potential between the two leads is abruptly applied.

It has been shown that in the long time limit, and provided the leads are infinite (more rigorously, provided they form a continuum of states), a steady-state is achieved, independent of the initial condition, with a current described by the Landauer Formula [13].

However, the current dynamics for finite leads has never been investigated. Such is the goal of this work. Hence, we study the time-evolution of the current in a prototypical 1D system, propagating in time the occupied quantum states of the system. Both partitioned and non-partitioned initial conditions are used and a comparison with the steady-state Landauer current is made. In both cases, the current was measured in different places of the central sample, for different disorder profiles and increasing lead sizes.

The paper is organized as follows: In Sect. 2 we introduce the model and essential definitions used for our numerical studies. In Sect. 3, we outline the basics of the method used to calculate the time-dependent current in this work and present our results. Finally, in Sect. 4, discuss our results and present conclusions.

\section{Model Hamiltonian and Local Current}

In order to study the current dynamics and the possible emergence of steady-state transport in a finite system, we will consider the prototypical one-dimensional tight-binding model, with nearest neighbor hoppings, represented in Fig. 1. This is described by the Hamiltonian:

$$
\mathcal{H}(t)=\sum_{n=0}^{L-1}\left(\epsilon_{n}^{\mathrm{d}}+v_{n}^{\mathrm{ext}}(t)\right) c_{n}^{\dagger} c_{n}-\sum_{n=0}^{L-2} w_{n}^{n+1}(t)\left(c_{n+1}^{\dagger} c_{n}+c_{n}^{\dagger} c_{n+1}\right) .
$$

The chain has $L$ sites, which we split into left and right leads, and a central sample. As depicted in Fig. 1, the left (right) lead, is formed by $L_{l}$ sites $-0 \leq n \leq L_{l}-1\left(L_{l}+L s \leq n \leq L-1\right)$ 
- and the central sample is formed by $L_{s}$ sites - $L_{l} \leq n \leq L_{l}+L_{s}-1$. In will consider the limit of very large leads $\left(L_{l} \gg L_{s}\right)$.

Sites in the central sample are subject to an on-site random disorder potential $\epsilon_{n}^{\mathrm{d}}$, uniformly sampled from $\left[-\frac{W}{2}, \frac{W}{2}\right]$. $w_{n}^{n+1}(t)$ are the nearest-neighbors hoppings with constant value $w$, except for the sample-lead bonds, and $v_{n}^{\text {ext }}(t)$ is an external bias potential, constant inside the leads (no electric field) and linear in the sample region (uniform electric field), as illustrated in Fig. 1. For $t>$ 0 , the potential profile is given by $V_{n}$, where:

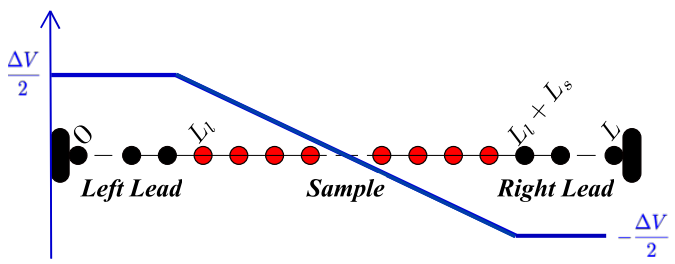

Figure 1. Prototype model used to simulate transport across an one-dimensional sample coupled to finite leads. The red dots are the places where there is a disordered potential and the blue curve is the profile of the externally applied potential. The chain has open boundaries.

$$
V_{n}=\mp \frac{e}{2} \Delta V \quad \text { if } n \in \text { Left (Right) Lead } \quad \text { and } \quad V_{n}=\frac{e}{2} \Delta V\left(1-2 \frac{n+L_{l}}{L_{s}}\right) \quad \text { if } n \in \text { Sample , }
$$

where $\Delta V$ measures the bias applied to the contacts. A similar scheme was already used in computer simulations by Poppescu et al [12] on a different context.

As referred in Sect. 1, we will consider two setups having different initial configurations and perturbations. Namely:

- Partitioned Setup: the sample and leads are initially disconnected, $w_{L_{l}-1}^{L_{l}}(t)=w_{L_{l}+L_{S}-1}^{L_{l}+L_{S}}(t)=$ $w \Theta(t)$, and the external bias is constant in time, $v_{n}^{\text {ext }}(t)=V_{n}$. The initial state of the system for $t<0$, is obtained by filling up eigenstates of the isolated leads and central sample, obtained from $\mathcal{H}(t<0)$, according to local chemical potentials: $E_{F} \pm \Delta V / 2$ for the left/right lead and $E_{F}$ for the central sample;

- Non-Partitioned Setup: the sample and leads are always connected, $w_{L_{l}-1}^{L_{l}}(t)=$ $w_{L_{l}+L_{S}-1}^{L_{l}+L_{S}}(t)=w$, and the bias is applied only for $t>0, v_{n}^{\text {ext }}(t)=\Theta(t) V_{n}$. The initial state of the connected system is obtained by filling up the eigenstates of the full system, obtained from $\mathcal{H}(t<0)$, according to a global chemical potential $E_{F}$.

Finally, the charge current flowing from site $n$ to site $n+1$, is described by the operator:

$$
\mathcal{I}_{n}^{n+1}=\frac{e w}{i \hbar}\left(c_{n+1}^{\dagger} c_{n}-c_{n}^{\dagger} c_{n+1}\right)
$$

\section{The Numerical Method and Results}

In this section, we outline the method used to numerically calculate the $I_{n}^{n+1}(t)$, after the external perturbation is suddenly connected. Let us suppose that $|\Psi(0)\rangle$ is some arbitrary occupied state. Then, its time-evolution is simply given by

$$
|\Psi(t)\rangle=e^{-i \mathcal{H}(t>0) t}|\Psi(0)\rangle,
$$

where $\mathcal{H}(t>0)$ is always time-independent and already includes the perturbation. Instead of calculating this exponential by diagonalization $\left(O\left(N^{3}\right)\right.$ effort), and since $\mathcal{H}$ has a bounded spectrum, we may expand it as a Chebyshev series [15], yielding

$$
|\Psi(t)\rangle=\sum_{n}^{\infty} \frac{2}{1+\delta_{n, 0}}(-i)^{n} J_{n}(\lambda t) T_{n}(\tilde{\mathcal{H}})|\Psi(0)\rangle,
$$


where $\tilde{\mathcal{H}}=\lambda^{-1} \mathcal{H}(t>0)$ is the rescaled Hamiltonian with spectrum inside ]-1, 1[. Now, the most expensive part of the computation lies in calculating $\left|\Psi_{n}\right\rangle=T_{n}(\tilde{\mathcal{H}})|\Psi(0)\rangle$, up to some truncation order $M$. This may be done recursively, using the well-known properties of the Chebyshev Polynomials, $T_{0}(x)=0, T_{1}(x)=x$ and $T_{n+1}(x)=x T_{n}(x)-T_{n-1}(x)$. Alternatively,

$$
\left|\Psi_{0}\right\rangle=|\Psi(0)\rangle, \quad\left|\Psi_{1}\right\rangle=\tilde{\mathcal{H}}|\Psi(0)\rangle \quad \text { and } \quad\left|\Psi_{n+1}\right\rangle=\tilde{\mathcal{H}}\left|\Psi_{n}\right\rangle-\left|\Psi_{n-1}\right\rangle .
$$

The series of Eq. 7 is seen to behave well upon truncation, as long as one keeps $M \gtrsim 4 \lambda t$, which makes the calculation of $|\Psi(t)\rangle$ an $O(t N)$ process, optimized by a minimal choice of $\lambda$. Finally, to calculate the local electric current as a function of time, for all $t>0$, we may use the previous method in the expectation value of $\mathcal{I}_{s}^{s+1}$. This yields

$$
I_{n}^{n+1}(t)=\sum_{\text {occupied } \alpha}\left\langle\psi_{\alpha}\left|e^{i \mathcal{H}(t>0) t} \mathcal{I}_{n}^{n+1} e^{-i \mathcal{H}(t>0) t}\right| \psi_{\alpha}\right\rangle
$$

where $\left|\Psi_{\alpha}\right\rangle$ is an initially occupied eigenstate of $\mathcal{H}(t<0)$. Furthermore, Eq. (9) may be recast in the more useful form

$$
I_{n}^{n+1}(t)=\frac{2 e w}{\hbar} \operatorname{Im} \sum_{\text {occupied } \alpha}\left\langle\phi_{n}(-t) \mid \Psi_{\alpha}\right\rangle\left\langle\Psi_{\alpha} \mid \phi_{n+1}(-t)\right\rangle,
$$

where $\left|\phi_{n}\right\rangle=c_{n}^{\dagger}|0\rangle$ is the state of an electron located at site $n$. The obvious advantage of the previous equation is that the numerical time-evolution need only to be performed twice.
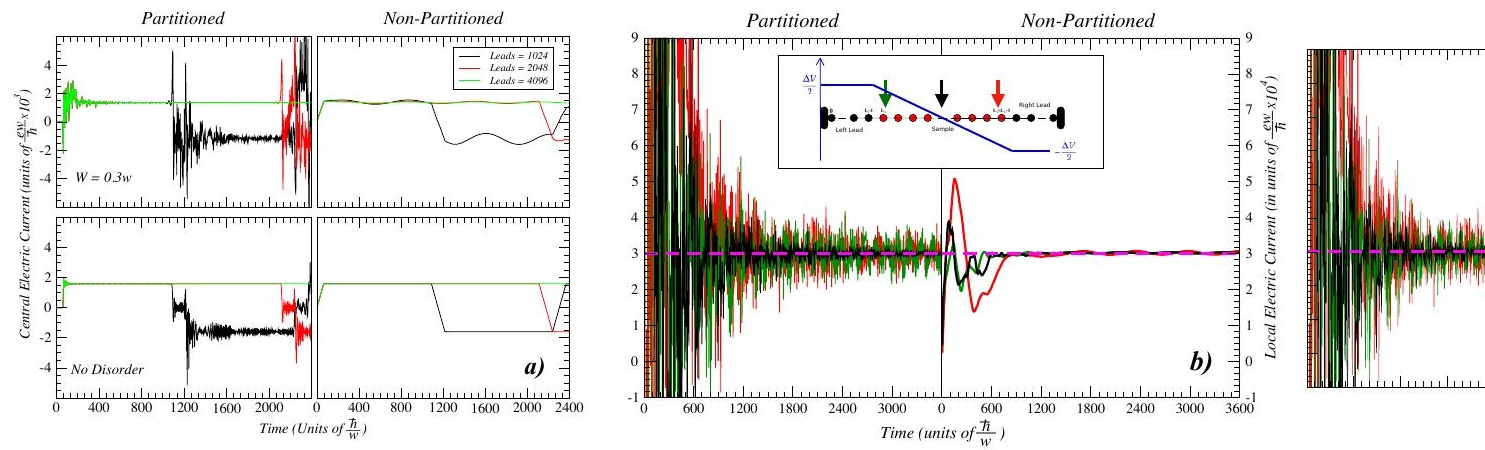

Figure 2. a) Plots of the time-dependent current across the central bond of a sample with $L_{S}=256$ (with and without disorder), in both setups and increasing lead sizes. $b$ ) Plots of the current measured in the central and boundaries bonds (see legend) of a disordered sample ( $W=0.5 \mathrm{w})$ with $L_{S}=512$ connected to leads with $L_{l}=2^{16}$ sites. Both setups are also compared and the dashed magenta line are the values of the Landauer current obtained for this sample. In $a$ ) and $b$ ) the bias used was $\Delta V=0.01 w$.

In Fig. 2 a), we show the current across the sample's center as a function of time, for both initial setups, with or without disorder. In the partitioned setup, the results are similar to the ones obtained earlier for a multi-level system by Pal et al [9], where a quasisteady state Landauer current is found after a short transient. The Landauer current was obtained by writing the transmission function in Eq. (1) as $\mathcal{T}(E)=\gamma_{L}(E) \gamma_{R}(E)\left|\mathcal{G}_{0, L_{s}+1}^{r}\right|^{2}$, with $\gamma_{L / R}(E)=$ $\sqrt{4 w^{2}-(E \mp e \Delta V / 2)}$ and the retarded Green's function of the specific sample, in contact with semi-infinite leads, is evaluated using the recursive green function algorithm [8, 17]. 
The main novelty is that the same current is now obtained using the non-partitioned setup, which numerically shows that, for large but finite leads, the quasisteady value of the current is independent of the initial conditions. Hence it agrees with the proof given in [13] for the case of infinite leads.

However, this quasisteady state is followed by a current inversion (perfect in the ordered case) at a time $t_{r}$. The reflection of the propagating wavefunctions at $E_{F}$ by the boundaries is conjectured to cause these inversions, seen in all plots of Fig. 2 a), which is confirmed by fact that $t_{r} \approx v_{F}^{-1} L_{l}$. Furthermore, since we do local measurements, we are also able to access the spacial profile of the current in the sample. In Fig. 2 b), we show that the quasisteady state current is indeed uniform, in the limit of very large leads and for both setups.

Nevertheless, we also report the existence of sample- and setup-specific oscillations of the quasisteady state current which are due to finite-size effects and disappear as $L_{l}$ and $t$ are increased. This is visible in the upper left panel of Fig. 2.

A definitive proof that a Landauer current is always established, for all biases, is provided by comparing the $I(V)$ curves of random samples as obtained from the quasisteady state current of our method, with the ones obtained from Eq. (1). The results are shown in Fig. 3, where one sees that all the previous examples were well beyond a linear transport regime.

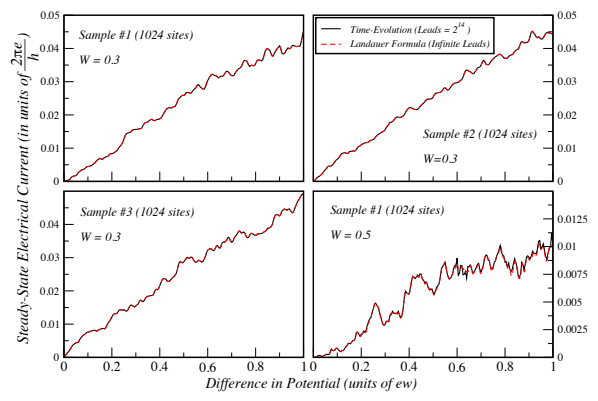

Figure 3. $I(\Delta V)$ curves of four disordered samples. The black curves were obtained from the quasisteady state regime of quantum dynamics in the non-partitioned setup with $L_{l}=2^{14}$ sites. The red dashed curves were obtained from the Landauer formula [Eq. (1)]. $E_{F}=0$ is assumed.

\section{Summary and Conclusions}

In this work, we studied the onset of quasisteady state transport regimes in large, but finite systems. Using a prototypical 1D tight-binding model, we have seen that the time-dependent current reaches a quasistationary value, even for finite leads. This quasisteady state regime, occurs at intermediate times, after transient dynamics have died out, but before the recurrent current inversions occur, due to the reflection of the electron wavefunctions at the boundaries.

As the size of the leads increases, so thus this recurrence time. During the quasisteady state regime, the current profile is nearly homogeneous as expected, and the value of the current in this quasisteady state regime coincides with the Landauer value, for large enough leads. Importantly, this quasisteady state regime is also independent on the initial condition of the system. We have thus extended previous results, valid only for infinite leads, on the emergence of a stationary Landauer current [3] and the independence of this state on the initial conditions [13] to systems with large, but finite leads.

Finally, the authors remark that these results and further developments on the timedependent particle transport with "finite leads" have been published recently in Santos Pires et al [11].

\section{Acknowledgements}

While doing this work, J.P.S.P. was supported by the MAP-fis PhD grant PD/BD/142774/2018 of Fundação da Ciência e Tecnologia (FCT), Portugal. B.A. also 
acknowledges financing from FCT through Project No CEECIND/02936/2017. The authors jointly acknowledge financing from FCT and COMPETE 2020 program in FEDER component (European Union), through the projects POCI-01-0145-FEDER-028887 and UID/FIS/04650/2013.

\section{References}

[1] A. Ferreira and E. R. Mucciolo. Phys. Rev. Lett., 115:106601, 2015.

[2] D. S. Fisher and P. A. Lee. Phys. Rev. B, 23(12):6851-6854, 1981.

[3] A.-P. Jauho, N. S. Wingreen, and Y. Meir. Phys. Rev. B, 50(8):5528-5544, 1994.

[4] S. M. João and J. M. Viana Parente Lopes. Journal of Physics: Condensed Matter, 32(12):125901, 2019.

[5] Simão M. João, Miša Anđelković, Lucian Covaci, Tatiana G. Rappoport, João M. V. P. Lopes, and Aires Ferreira. Royal Society Open Science, 7(2):191809, 2020.

[6] R. Kubo. J. Phys. Soc. Japan, 12(6):570-586, 1957.

[7] R. Landauer. Phil. Mag., 21(172):863-867, 1970.

[8] A. MacKinnon. Z. Phys. B: Cond. Matt., 59(4):385-390, 1985.

[9] P. P. Pal, S. Ramakrishna, and T. Seideman. Jour. Chem. Phys., 148(14):144707, 2018.

[10] D. J. Passos, G. B. Ventura, J. M. Viana Parente Lopes, J. M. B. Lopes dos Santos, and N. M. R. Peres. 97(23):235446, 2018.

[11] J. P. Santos Pires, B. Amorim, and J. M. Viana Parente Lopes. Landauer transport as a quasisteady state on finite chains under unitary quantum dynamics. arXiv:1909.12402 (Accepted in Phys. Rev. B), 2020.

[12] B. S. Popescu and A. Croy. Phys. Rev. B, 95(23):235433, 2017.

[13] G. Stefanucci and C.-O. Almbladh. Phys. Rev. B, 69(19):195318, 2004.

[14] A. D. Stone and A. Szafer. IBM Jour. Res. Develop., 32(3):384-413, 1988.

[15] H. Tal-Ezer and R. Kosloff. Jour. Chem. Phys., 81(9):3967-3971, 1984.

[16] G. B. Ventura, D. J. Passos, J. M. B. Lopes dos Santos, J. M. Viana Parente Lopes, and N. M. R. Peres. Phys. Rev. B, 96:035431, 2017.

[17] Michael Wimmer. Quantum transport in nanostructures: from computational concepts to spintronics in graphene and magnetic tunnel junctions. Number 5 in Dissertationsreihe der Fakultät für Physik der Universität Regensburg. Univ.-Verl. Regensburg, 2009. 